\title{
Overcoming immunoregulatory plasticity of mesenchymal stem cells for accelerated clinical applications
}

\author{
Nayoun Kim $^{1,2} \cdot$ Seok-Goo Cho ${ }^{1,2,3}$ (I)
}

Received: 19 November 2015 / Revised: 25 November 2015 / Accepted: 30 November 2015 / Published online: 11 December 2015

(C) The Japanese Society of Hematology 2015

\begin{abstract}
Mesenchymal stem cells (MSCs) are multipotent stromal cells with the potential to differentiate into different tissue lineages. In addition to their differentiation potential, MSCs possess immunomodulatory properties that have created growing interest in both pre-clinical and clinical research. Over the years, MSCs have been applied rapidly in the clinic in a wide variety of immune-mediated disorders; however, MSC therapy has shown contradictory results, often with poor clinical outcomes. Recently, studies on MSC-based immune modulation have provided possible explanations for the conflicting clinical reports. It is now generally recognized that the immunomodulatory properties of MSCs are not constitutive but are induced by various mediators present in the inflammatory microenvironment. Different inflammatory stimuli are able to polarize MSCs to elicit distinct immunomodulatory phenotypes. Thus, the concepts of plasticity and polarization of MSC-based immune modulation may have important therapeutic implications in the clinic. In this review, we focus on the underlying mechanisms of MSC-mediated immune regulation that contribute to their therapeutic potential. Importantly,
\end{abstract}

Seok-Goo Cho

chosg@catholic.ac.kr

1 Institute for Translational Research and Molecular Imaging, Seoul St. Mary's Hospital, The Catholic University of Korea College of Medicine, \#505, Banpo-Dong, Seocho-Ku, Seoul 137-040, Korea

2 Laboratory of Immune Regulation, Convergent Research Consortium for Immunologic Disease (CRCID), Seoul, Korea

3 Department of Hematology, Catholic Blood and Marrow Transplantation Center, Seoul St. Mary's Hospital, The Catholic University of Korea College of Medicine, Seoul, Korea we discuss novel strategic approaches that enhance the therapeutic potential of MSCs through a consideration of MSC plasticity in immune modulation.

Keywords Mesenchymal stem cells · Polarization · Plasticity $\cdot$ Immune modulation $\cdot$ Clinical application

\section{Introduction}

Mesenchymal stem cells (MSCs) were originally described by Friedenstein and colleagues as adult bone marrowderived stromal stem cells with the potential to differentiate into adipogenic, chondrogenic, and osteogenic lineages $[1,2]$. Since then, MSCs have been isolated and expanded from a wide variety of tissue types, and the use of MSCs has accelerated in both research and clinical labs. To address the inconsistent characterization of MSCs by different investigators, the International Society for Cellular Therapy provided minimal criteria for defining MSCs, which included adherence to plastic, specific surface antigen expression, assessment by flow cytometry, and multipotent differentiation potential under standard in vitro conditions [3]. Surface antigen expression of MSCs includes the expression of mature stromal cell markers (CD105, CD73, and CD90) and the absence of hematopoietic and endothelial markers (CD45, CD34, CD14 or CD11b, CD79a or CD19) and human leukocyte antigen-DR.

Early pre-clinical studies on MSCs focused on the role of MSCs in tissue regeneration due to their ability to differentiate and migrate to sites of injury $[4,5]$. Contrary to initial expectations, MSCs showed low engraftment rates and short life spans, suggesting that differentiation-independent mechanisms played a more important role in the therapeutic effects of MSCs [6, 7]. Increasing observations 
revealed that MSCs produced large amounts of cytokines, growth factors, and differentiation factors involved in the regulation of inflammation and immune responses $[8,9]$. Thus, the therapeutic potential exerted by MSCs depends largely on their capacity to secrete soluble mediators that influence various immune cell types and factors involved in the microenvironment. Based on these data, numerous clinical studies have sought to exploit the immunoregulatory properties of MSCs in various pathological conditions over the years [10]. With increasing knowledge of MSCmediated immune modulation, numerous investigators now report that the immunomodulatory activities of MSCs are not constitutive but are induced by different inflammatory mediators present in the microenvironment [11-14], and depending on the inflammatory stimuli, MSCs show high functional plasticity, in that MSCs not only inhibit immune responses but also enhance them. Recently, the concept of MSC plasticity in immune modulation has provided some explanation for the contradictory and often discouraging outcomes of past and ongoing MSC-based clinical experiences. Understanding MSC plasticity has provided a new paradigm for MSC-based therapy and may additionally accelerate the clinical application of MSCs.

In this review, we provide an overview of the basic concepts of the immunomodulatory properties of MSCs and highlight the latest findings on plasticity and the polarization of MSCs involved in immune regulation. Finally, we discuss future considerations for the clinical application of MSCs to improve their therapeutic efficacy.

\section{Immunomodulatory effects of MSCs}

Initial observations that MSCs interact closely with the bone marrow environment and that MSCs express the adhesion molecules VCAM-1, ICAM-1, and LFA-3 involved in T-cell interactions resulted in the discovery of the immunomodulatory properties of MSCs [15]. Subsequently, numerous studies reported potent immunosuppressive effects of MSCs, mediated primarily by paracrine effects. MSCs produce a broad spectrum of growth factors and cytokines, and the full extent of immunosuppressive factors remains to be determined. Major soluble factors involved in immune modulation include transforming growth factor (TGF)- $\beta$, heme oxygenase, insulin like growth factors, interleukin (IL)-6, prostaglandin E2, and indoleamine 2,3-dioxygenase (IDO). Additionally, some studies have reported direct cell-to-cell contact mechanisms involved in MSC-mediated immune modulation [8, 16].

MSCs interact and act on lymphocytes in both innate and adaptive immune responses [8,17]. Within the innate immune system, MSCs inhibit the activation of pro-inflammatory monocytes and macrophages and promote the conversion of pro-inflammatory M1 macrophages into antiinflammatory M2 macrophages [18, 19]. In addition, MSCs inhibit the differentiation of monocytes into mature dendritic cells (DCs) [20]. Antigen-presenting DCs may also be converted into anti-inflammatory tolerogenic DCs in the presence of MSCs. Tolerogenic DCs produce high levels of IL-10 and have reduced ability to stimulate T-cell proliferation. Moreover, MSCs play a fundamental role in the regulation of natural killer cell proliferation and their effector cytotoxic functions [21]. In the adaptive immune system, MSCs suppress T-cell proliferation directly and activation, and also regulate the differentiation of helper $\mathrm{T}(\mathrm{Th})$ cells $[15,22,23]$. MSCs suppress both pro-inflammatory Th1 and Th17 cell subsets while promoting anti-inflammatory Th2 cell subsets. Importantly, MSCs promote the differentiation of functional regulatory T cells (Tregs) from naïve Th0 cells [24], a unique T-cell subpopulation that specializes in suppressing immune responses. Tregs induced in the presence of MSCs are capable of potent inhibitory functions against effector cell proliferation. Finally, MSCs are capable of inhibiting B-cell proliferation through direct cell-to-cell contact. Furthermore, MSCs inhibit effector B cell functions, including plasma cell differentiation and immunoglobulin production [25].

\section{Limitations of MSC-mediated immune modulation in the clinical setting}

With knowledge of the immunosuppressive functions of MSCs, the first clinical application of MSC therapy was in a steroid-refractory graft-versus-host disease (GVHD) patient following bone marrow transplantation (BMT) who was initially unresponsive to all conventional therapies [26]. The dramatic clinical improvement in this patient by MSC treatment led to a series of phase I/II multicenter trials by the European Group for BMT [27], which also showed promising results for the treatment of GVHD. However, several studies demonstrated mixed results regarding MSC efficacy, depending on the age, organ involvement, and severity of GVHD patients, suggesting that MSC therapy may be more effective in specific environments [17]. For example, a greater proportion of pediatric than adult patients responded to MSCs, and better outcomes of MSC therapy were observed in steroid-refractory acute GVHD compared with de novo GVHD or chronic GVHD patients [28, 29]. However, in a randomized placebo-controlled clinical trial by Osiris Therapeutics using MSCs for the treatment of steroid-refractory GVHD, public reports stated that MSCs failed to demonstrate therapeutic efficacy compared with placebo controls [30]. Thus, despite the considerable progress in MSC treatment that had been made over the years, subsequent clinical studies produced ambiguous results regarding the therapeutic efficacy of MSCs. 
In addition to GVHD, MSC therapy has been explored in trials of various immune-mediated conditions, such as cardiovascular disease, Crohn's disease, osteoarthritis, rheumatoid arthritis, and amyotrophic lateral sclerosis (ALS) [17, 31]. However, investigators struggled to show clear therapeutic efficacy in these chronic immune disorders. In Crohn's disease, therapeutic efficacy of MSC therapy through intralesional infusions has been demonstrated by local healing of Crohn's fistulas [32]. However, systemic infusions of MSCs did not inhibit progression of the disease [33]. In osteoarthritis patients, MSC therapy showed promising results with improvements in clinical parameters, including pain and function, compared with baseline. However, when the results were compared with historical control groups, all clinical scores were significantly poorer in the MSC-treated group [34]. Recently, MSC therapy has also been applied to RA patients; the delay in clinical application was associated with conflicting results from pre-clinical studies. In refractory RA patients, MSC therapy induced a short-term reduction in clinical scores, but the clinical improvement was not maintained during the follow-up period; thus, complete remission was not achieved in any patient [35]. In a larger study, MSC therapy provided clinical efficacy in active RA patients for at least 3 months, and additional MSC administrations at 3 months stabilized the disease [36]. Furthermore, in a recent study of MSC therapy in ALS patients, it was demonstrated that immunomodulatory factors secreted by MSCs were predictive of the effectiveness of MSC therapy and could potentially identify the patients responsive to therapy. Although the clinical outcomes following MSC therapy did not differ significantly from those of the control groups, ALS patients who responded to MSC therapy showed enhanced levels of immunomodulatory factors, such as VEGF and TGF- $\beta$, in the remnant MSC specimens following administration, compared with non-responders [37].

Thus, these conflicting observations on MSC therapy in various diseases suggest that to achieve the well-defined therapeutic benefits of MSC therapy, mechanisms involved in MSC-mediated immune modulation need to be investigated further.

\section{Plasticity of MSC-mediated immune modulation}

Pre-clinical studies of MSC therapy have highlighted that MSCs actively sense and react to their environment. The potent immunomodulatory properties exerted by MSCs are not constitutively immunosuppressive but are induced by environmental inflammatory mediators. Currently, the plasticity of MSC-mediated immune modulation represents a new paradigm in MSC immunobiology.

\section{Licensing of MSCs by inflammatory stimuli}

MSCs require exposure to pro-inflammatory cytokines, such as IFN- $\gamma$, TNF- $\alpha$, IL- $1 \alpha$, and IL- $1 \beta$, to activate MSCmediated immune suppressive effects. The inhibitory effects of MSCs on T-cell proliferation require the presence of IFN- $\gamma$, and exogenous addition of IFN- $\gamma$ can further enhance the suppressive activity of MSCs [38] through increased secretion of chemokine receptor ligands and immunosuppressive IDO. The role of IFN- $\gamma$ in MSC-mediated immune suppression has also been described in vivo in a GVHD model [39]. The therapeutic effects of MSCs correlated strongly with the presence of IFN- $\gamma$ following the development of GVHD. Furthermore, when IFN- $\gamma$ knockout mice were used as donors for GVHD, MSC treatment could not control the disease [39]. Pretreatment of MSCs with IFN- $\gamma$, however, restored the immunoregulatory capacities of the MSCs.

Macrophages play a critical role in vivo in initiating and regulating the immunomodulatory effects of MSCs. Depending on the inflammatory stimuli, monocytes develop into "classical" M1 macrophages or "alternatively activated" M2 macrophages. During acute inflammatory responses, Th1 cytokines induce the polarization of M1 macrophages that secrete high levels of IFN- $\gamma$ and TNF$\alpha$, involved in MSC licensing. In contrast, during chronic inflammation, high levels of Th2 cytokines polarize M2 macrophages that secrete IL-6 and IL-10, resulting in alternative licensing of MSCs [40].

\section{Immunoregulatory fate of MSCs following licensing}

MSCs that respond to different inflammatory stimuli are capable of differential polarization $[11,14]$ (Table 1; Fig. 1). Following tissue injury, inflammatory mediators such as cytokines and Toll-like receptors (TLR) agonists promote MSCs to release various chemokines, such as CXCL-9 and CXCL-10, and adhesion molecules, including intercellular adhesion molecule-1 and vascular cell adhesion molecule-1, which subsequently recruit lymphocytes to the inflammatory site $[23,41,42]$.

When MSCs encounter lymphocytes in the microenvironment, NO and IDO play key roles in orchestrating MSCs in immune modulation [43]. The production of NO and IDO by MSCs has been demonstrated to be involved in the immunosuppressive effects on various lymphocytes. Likewise, inhibition of the NO and IDO pathways of MSCs dramatically reduces the suppressive effects of MSCs on lymphocytes [23]. During acute inflammation, when M1 macrophages are present and levels of IFN- $\gamma$ and TNF- $\alpha$ are high, MSCs are licensed to acquire an immunosuppressive phenotype, in which high levels of NO and IDO are produced. Robust production of NO and IDO by activated 
Table 1 Factors involved in MSC-mediated immune modulation

\begin{tabular}{llll}
\hline $\begin{array}{l}\text { Pro-inflammatory stimuli for } \\
\text { licensing }\end{array}$ & $\begin{array}{l}\text { Secreted chemokines that recruit } \\
\text { lymphocytes }\end{array}$ & $\begin{array}{l}\text { Secreted immunosuppressive } \\
\text { molecules }\end{array}$ & $\begin{array}{c}\text { Immunoregulatory effects on } \\
\text { lymphocytes }\end{array}$ \\
\hline IFN- $\gamma$ & TGF- $\beta$ & $\begin{array}{c}\text { Inhibit activation of pro-inflamma- } \\
\text { tory monocytes } \\
\text { Induce anti-inflammatory M2 } \\
\text { macrophages }\end{array}$ \\
IL-1 $\alpha$ & CXCL-9 $\alpha$ & Heme oxygenase & $\begin{array}{c}\text { Inhibit differentiation of monocytes } \\
\text { into DCs }\end{array}$ \\
IL-1 $\beta$ & CXCL-10 & Induce tolerogenic DCs \\
TLR3 priming & CCL5 & IL-6 & $\begin{array}{c}\text { Inhibit proliferation and cytotoxic- } \\
\text { ity of NK cells }\end{array}$ \\
TLR4 priming & PGE2 & $\begin{array}{c}\text { Suppress T-cell proliferation and } \\
\text { activation }\end{array}$ \\
& & IDO & Induce Tregs \\
\end{tabular}

$C C L$ CC chemokine ligand, $C X C L \mathrm{C}-\mathrm{X}$-C motif ligand, $D C$ dendritic cell, $I D O$ indoleamine 2,3-dioxygenase, $I F N$ interferon, $I G F$ insulin like growth factor, $I L$ interleukin, $N K$ natural killer, $N O$ nitric oxide, $P G E$ prostaglandin, $T G F$ transforming growth factor, $T L R$ toll-like receptor, $T N F$ tumor necrosis factor, Treg regulatory $\mathrm{T}$ cell

MSCs promote the inhibition of highly proliferating effector cells involved in the pathophysiological process of disease. However, during chronic inflammation, when M2 macrophages are present and levels of IFN- $\gamma$ and TNF- $\alpha$ are relatively low, MSCs are alternatively licensed to produce reduced levels of NO and IDO. Due to insufficient levels of NO and IDO, MSCs fail to control the immune response elicited by effector lymphocytes. While these MSCs produce low levels of immunosuppressive NO and IDO, MSCs maintain production of chemokines, such as CXCL9, CXCL10, RANTES, MIP1- $\alpha$, and MIP- $\beta$, and thus continue to recruit and enhance effector $\mathrm{T}$ cells. Thus, MSCs polarized in such environments are often characterized as immune enhancing.

Moreover, it has been recently demonstrated that the activation of TLRs present in MSCs, including TLR3 and TLR4, induce MSCs to acquire specific immunomodulatory functions [42]. TLR3 ligation by viral double-stranded RNA induces the polarization of immunosuppressive MSCs that produce high levels of NO and IDO. However, the activation of TLR4 by lipopolysaccharide promotes alternatively licensed MSCs that produce low levels of IDO and NO.

\section{Clinical relevance of MSC plasticity}

Although further investigations into the immunoregulatory mechanisms of MSC therapy and additional clinical experience are necessary, it is becoming evident that appropriate inflammatory stimuli determine the immunosuppressive fate of MSCs. In the clinical setting, varying inflammatory conditions in different immune-mediated disorders may result in different responses to MSC treatment. Cytokine profiles that are dominant during the acute phases of inflammation may differ from those of chronic disorders and, thus, differentially license MSCs. Furthermore, the inflammatory statuses that continue to fluctuate throughout the course of pathogenesis, and immune responses potentially alter the effects of MSC-mediated immune modulation. Importantly, most patients treated with MSC therapy concurrently receive high doses of immunosuppressants that alter the inflammatory profile in the microenvironment. Thus, in addition to the direct effects of immunosuppressants on MSCs, the effects of immunosuppressants on the local inflammatory environment and how they influence the functional plasticity of MSCs need to be considered.

\section{Novel strategies for accelerated clinical application of MSCs}

Today, the insights we have gained from studies on the plasticity of MSC-mediated immune modulation provide important therapeutic implications [14]. Here, we discuss novel strategic approaches to overcome the plasticity of MSCs for accelerated clinical applications (Fig. 2).

\section{Gene-modified MSCs}

Ideally, sustained production of immunomodulatory factors, independent of inflammatory stimuli, can significantly enhance the potency of MSCs. Pre-clinical studies have consistently demonstrated that intentional overexpression of anti-inflammatory genes, including HGF [44], IL-4 [45], 

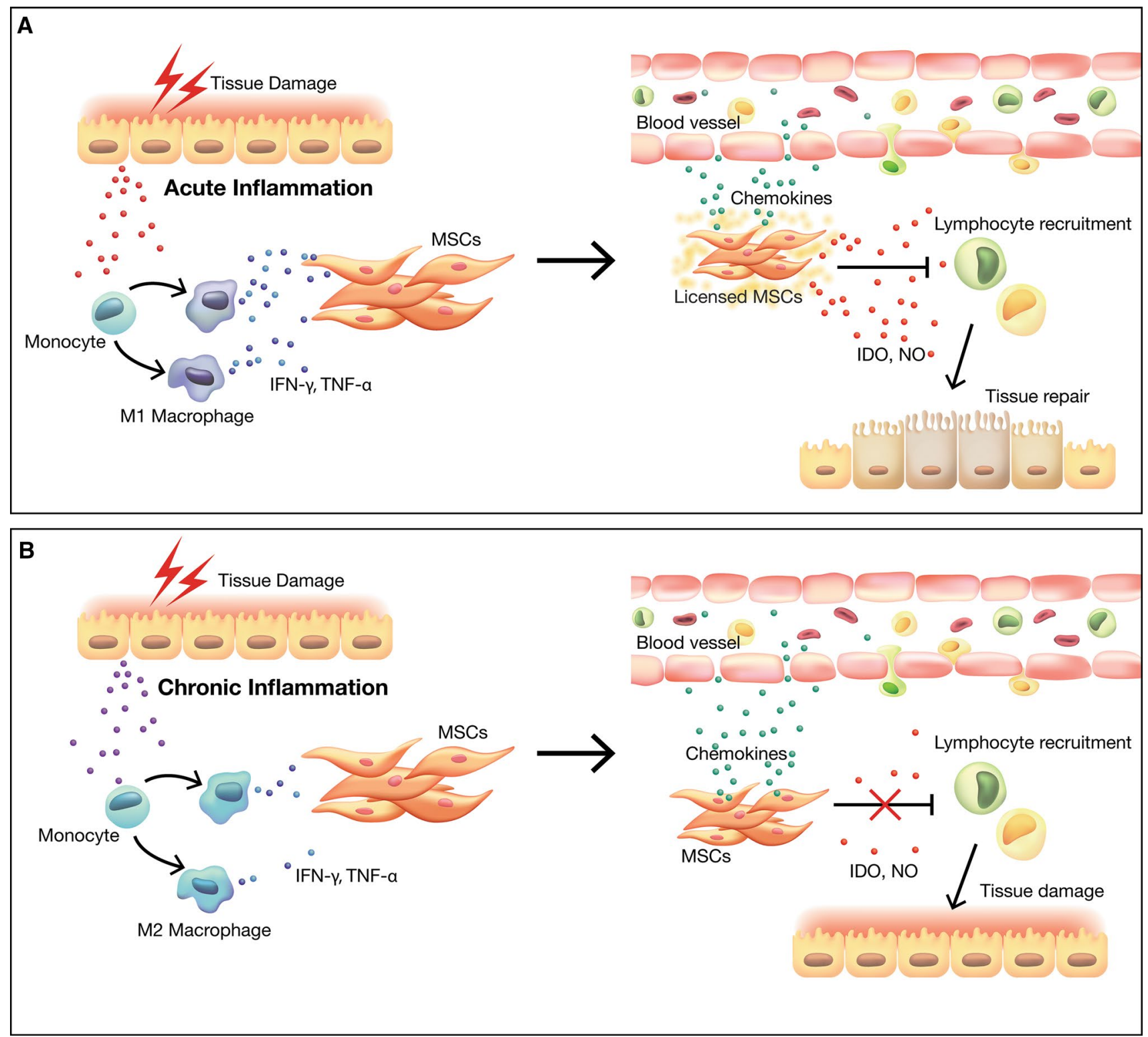

Fig. 1 Plasticity of MSCs in immune modulation. a At a site of tissue damage, local inflammatory mediators are secreted to activate monocytes into pro-inflammatory M1 macrophages. M1 macrophages produce high levels of pro-inflammatory cytokines, including IFN- $\gamma$ and TNF- $\alpha$, and license MSCs to acquire an immunosuppressive phenotype. In response to pro-inflammatory stimuli, activated MSCs produce chemokines that attract lymphocytes to the site of injury. Licensed MSCs produce high levels of the immunosuppressive molecules IDO and NO, which suppress the effector functions of recruited

IFN- $\gamma$ [46], IL-10 [47], and TGF- $\beta$ [48], could dramatically improve the clinical course in both acute and chronic immune-mediated disorders. However, the involvement of genetic manipulation raises important safety issues for use in the clinical setting, and thus progress towards clinical lymphocytes. As a result, the overall immune response is attenuated and tissue repair induced. b However, chronic inflammation induces the differentiation of monocytes into $\mathrm{M} 2$ macrophages that produce relatively low levels of IFN- $\gamma$ and TNF- $\alpha$. Chemokine production by MSCs is maintained and recruits lymphocytes to the site of inflammation. However, low levels of IFN- $\gamma$ and TNF- $\alpha$ provide insufficient immunosuppressive licensing of MSCs. As a result, reduced levels of IDO and NO are secreted by MSCs, in which uncontrolled lymphocytes aggravate the inflammatory immune response

applications has been relatively slow despite the therapeutic potential. Furthermore, there are concerns that overproduction of anti-inflammatory cytokines in gene-modified MSCs may paradoxically cause pathological immune responses in disease progression. 
Fig. 2 Novel approaches to MSC-based therapy. The concept of MSC plasticity has provided important therapeutic implications to accelerate the clinical applications of MSCs. The use of gene-modified MSCs, pre-conditioning of MSCs with licensing stimuli, the use of MSC-based combination cell therapy, and the establishment of standardized immunological characterization of MSCs may be considered in future studies

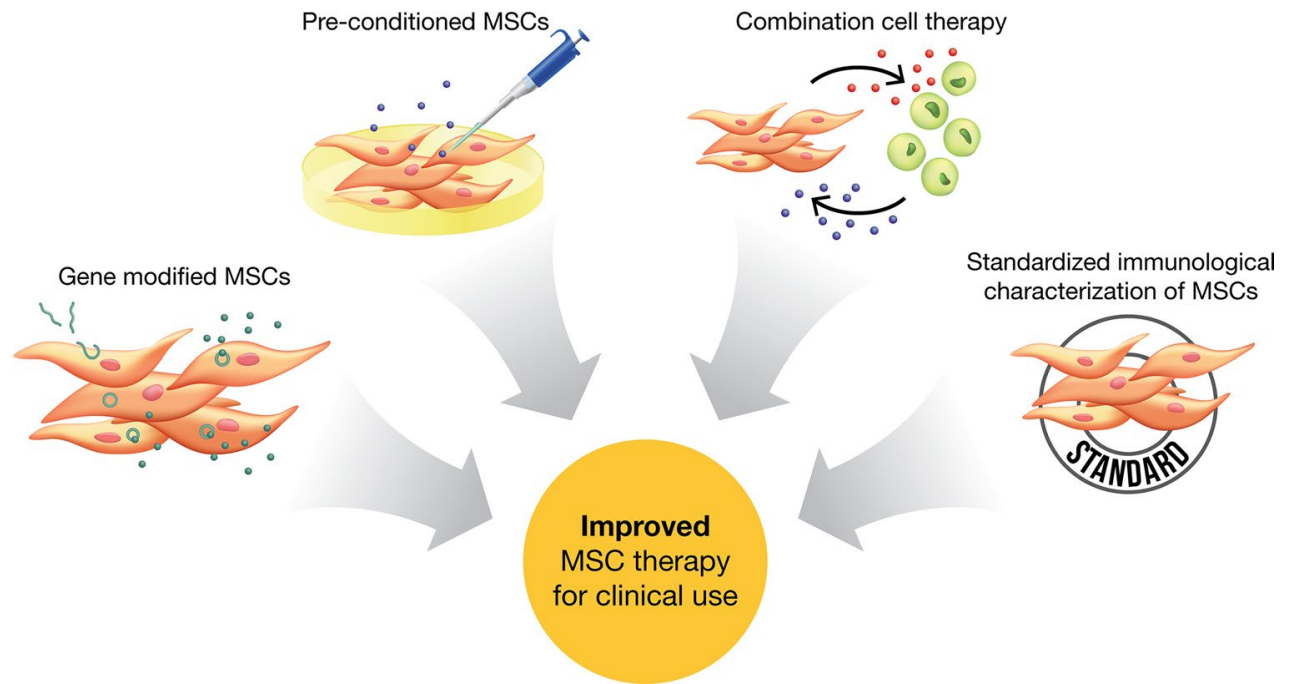

\section{Pre-conditioning of MSCs}

To overcome the limitations of gene-modified MSCs, ex vivo preconditioning of MSCs for transient immunosuppressive enhancements may be more relevant clinically. Pre-activation of MSCs with pro-inflammatory cytokines was initially reported in an experimental GVHD model using IFN- $\gamma$ [39]. It has been reported that the ex vivo pre-treatment of MSCs with cytokines, such as IFN- $\gamma$ [49] and TNF- $\alpha$ [50], resulted in enhanced immunosuppressive effects of MSCs. Other methods, such as hypoxia preconditioning [51] and activation of the nucleotide-binding oligomerization domain [52] expressed on MSCs, have been suggested. However, numerous other possibilities for pre-conditioning MSCs exist, including different concentrations of cytokines, different combinations of stimulating factors, and varied culture durations. Thus, it is essential to obtain standardized and optimized protocols for pre-conditioning MSCs that trigger efficient immunomodulatory effects.

\section{Combined cell-based immune modulation}

One of the major immunomodulatory effects of MSCs is the induction of Tregs, both in vitro and in vivo. MSCs promote the differentiation of Tregs that directly contribute to suppressing the immune response. In addition, the induction of Tregs may beneficially support the immunosuppressive activities of MSCs. Thus, close interactions and empowering relationships between MSCs and Tregs suggest a synergistic therapeutic effect when used together. In our group, we implemented the use of a combined cell therapy approach for the treatment of acute GVHD [53], induction of mixed chimerism following BMT [54], and prevention of allogeneic skin-graft rejection [55]. We observed that the combined cell therapy groups in all models showed enhanced survival and reduced clinicopathological symptoms. The combination of MSCs and Tregs could efficiently inhibit both Th1 and Th17 responses while promoting Treg levels in vivo, compared with single cell therapy groups. Importantly, deficient levels of circulating and local Tregs are characteristic of many pathologies. In a BMT model, there was a temporal gap following a myeloablative conditioning regimen until endogenous Tregs were reconstituted [53]. At this point, even pre-activated MSCs may show reduced immunomodulatory effects, because Tregs are not present. Similarly, in disease models that involve a dysregulated balance of Tregs, MSCs may not be able to exert their immunoregulatory activity fully. Thus, the co-administration of Tregs may compensate for insufficient levels of endogenous Tregs in the microenvironment. Moreover, we have recently discovered that the combination of MSCs and Tregs not only increases the repopulation of endogenous Tregs but also induces long-term survival and the stability of transferred Treg cells [56]. Finally, other immune cells, such as IL-10-producing regulatory $\mathrm{T}$ cells or DCs, may be considered in future studies.

\section{Standardized immunological characterization of MSCs prior to treatment}

In 2006, the MSC committee of the International Society of Cell Therapy (ISCT) established minimal criteria for characterizing MSCs [3]. However, immunomodulatory properties of MSCs were not considered, because the main objective at the time was to provide standardized cell preparations. Since then, a new working proposal has been put forward for the immunological characterization of MSCs. Due to variable culture conditions, such as culture medium, cell density, and additional growth factors 
used, the immunological characterization of the final MSC product may differ, and thus, implementing standard functional assessments to assess the immunomodulatory effects of MSCs prior to use may be beneficial [57]. Functional assays that investigate the immune regulatory response between resting and licensed MSCs may be informative prior to clinical use. The ISCT committee suggested MSC licensing assays using IFN- $\gamma$ with or without TNF- $\alpha$. Flow cytometric analyses characterizing the immunophenotypes of MSCs may be implemented as part of product characterization. While resting MSCs lack co-stimulatory molecules and major histocompatibility complex (MHC) expression, IFN- $\gamma$-treated MSCs upregulate various markers, including MHC I and II, cytokine-chemokine receptors, and adhesion molecules involved in immune regulation. In addition, MSCs produce high levels of IDO in response to IFN- $\gamma$, which plays a key role in lymphocyte inhibition. Thus, assessment of the IDO response should be central to functional assessments. Finally, well-designed and reproducible animal disease models are needed to validate the immunosuppressive properties of MSCs under different in vivo conditions prior to clinical use.

\section{Conclusions}

To conclude, the immunomodulatory properties of MSCs have created growing interest in various fields of medicine over the years. Recent studies have highlighted the plasticity of MSCs involved in immune regulation and revealed new insights into MSC-based immune modulation. Currently, new therapeutic guidelines to enhance MSC-based therapy have been suggested on the basis of in vitro observations. However, further in vivo studies will be necessary to validate the underlying mechanisms of MSCs in immune modulation. With the changes in the paradigm of MSC immunomodulation, it is anticipated in the near future that improved designs of MSC-based therapies will be implemented in the clinic.

Acknowledgments This study was supported by a Grant (HI14C3417) from the Korea Healthcare Technology R\&D Project, the Ministry for Health, Welfare, and Family Affairs, Republic of Korea, by a research fund of Seoul St. Mary's Hospital, The Catholic University of Korea, and by a grant from the Catholic Institute of Cell Therapy. The English in this document has been checked by at least two professional editors, both native speakers of English. For a certificate, please see: http://www.textcheck.com/certificate/rN8FVy.

\section{References}

1. Friedenstein AJ, Chailakhyan RK, Latsinik NV, Panasyuk AF, Keiliss-Borok IV. Stromal cells responsible for transferring the microenvironment of the hemopoietic tissues. Cloning in vitro and retransplantation in vivo. Transplantation. 1974;17:331-40.
2. Pittenger MF, Mackay AM, Beck SC, Jaiswal RK, Douglas R, Mosca JD, et al. Multilineage potential of adult human mesenchymal stem cells. Science. 1999;284:143-7.

3. Dominici M, Le Blanc K, Mueller I, Slaper-Cortenbach I, Marini F, Krause D, et al. Minimal criteria for defining multipotent mesenchymal stromal cells. The International Society for Cellular Therapy position statement. Cytotherapy. 2006;8:315-7.

4. Han F, Wang CY, Yang L, Zhan SD, Zhang M, Tian K. Contribution of murine bone marrow mesenchymal stem cells to pancreas regeneration after partial pancreatectomy in mice. Cell Biol Int. 2012;36:823-31.

5. Qian H, Yang H, Xu W, Yan Y, Chen Q, Zhu W, et al. Bone marrow mesenchymal stem cells ameliorate rat acute renal failure by differentiation into renal tubular epithelial-like cells. Int J Mol Med. 2008;22:325-32.

6. Lee RH, Pulin AA, Seo MJ, Kota DJ, Ylostalo J, Larson $\mathrm{BL}$, et al. Intravenous hMSCs improve myocardial infarction in mice because cells embolized in lung are activated to secrete the anti-inflammatory protein TSG-6. Cell Stem Cell. 2009;5:54-63.

7. von Bahr L, Batsis I, Moll G, Hagg M, Szakos A, Sundberg B, et al. Analysis of tissues following mesenchymal stromal cell therapy in humans indicates limited long-term engraftment and no ectopic tissue formation. Stem Cells. 2012;30:1575-8.

8. Nauta AJ, Fibbe WE. Immunomodulatory properties of mesenchymal stromal cells. Blood. 2007;110:3499-506.

9. Madrigal M, Rao KS, Riordan NH. A review of therapeutic effects of mesenchymal stem cell secretions and induction of secretory modification by different culture methods. J Transl Med. 2014;12:260.

10. Kim N, Cho SG. Clinical applications of mesenchymal stem cells. Korean J Intern Med. 2013;28:387-402.

11. Bernardo ME, Fibbe WE. Mesenchymal stromal cells: sensors and switchers of inflammation. Cell Stem Cell. 2013;13:392-402.

12. Ma S, Xie N, Li W, Yuan B, Shi Y, Wang Y. Immunobiology of mesenchymal stem cells. Cell Death Differ. 2014;21:216-25.

13. Shi Y, Su J, Roberts AI, Shou P, Rabson AB, Ren G. How mesenchymal stem cells interact with tissue immune responses. Trends Immunol. 2012;33:136-43.

14. Wang Y, Chen X, Cao W, Shi Y. Plasticity of mesenchymal stem cells in immunomodulation: pathological and therapeutic implications. Nat Immunol. 2014;15:1009-16.

15. Bartholomew A, Sturgeon C, Siatskas M, Ferrer K, McIntosh $\mathrm{K}$, Patil S, et al. Mesenchymal stem cells suppress lymphocyte proliferation in vitro and prolong skin graft survival in vivo. Exp Hematol. 2002;30:42-8.

16. Stagg J, Galipeau J. Mechanisms of immune modulation by mesenchymal stromal cells and clinical translation. Curr Mol Med. 2013;13:856-67.

17. Kim N, Im KI, Lim JY, Jeon EJ, Nam YS, Kim EJ, et al. Mesenchymal stem cells for the treatment and prevention of graftversus-host disease: experiments and practice. Ann Hematol. 2013;92:1295-308.

18. Kim J, Hematti P. Mesenchymal stem cell-educated macrophages: a novel type of alternatively activated macrophages. Exp Hematol. 2009;37:1445-53.

19. Maggini J, Mirkin G, Bognanni I, Holmberg J, Piazzon IM, Nepomnaschy I, et al. Mouse bone marrow-derived mesenchymal stromal cells turn activated macrophages into a regulatory-like profile. PLoS One. 2010;5:e9252.

20. Spaggiari GM, Abdelrazik H, Becchetti F, Moretta L. MSCs inhibit monocyte-derived DC maturation and function by selectively interfering with the generation of immature DCs: central role of MSC-derived prostaglandin E2. Blood. 2009;113:6576-83. 
21. Spaggiari GM, Capobianco A, Abdelrazik H, Becchetti F, Mingari MC, Moretta L. Mesenchymal stem cells inhibit natural killer-cell proliferation, cytotoxicity, and cytokine production: role of indoleamine 2,3-dioxygenase and prostaglandin E2. Blood. 2008;111:1327-33.

22. Keating A. How do mesenchymal stromal cells suppress $\mathrm{T}$ cells? Cell Stem Cell. 2008;2:106-8.

23. Ren G, Zhang L, Zhao X, Xu G, Zhang Y, Roberts AI, et al. Mesenchymal stem cell-mediated immunosuppression occurs via concerted action of chemokines and nitric oxide. Cell Stem Cell. 2008;2:141-50.

24. English K, Ryan JM, Tobin L, Murphy MJ, Barry FP, Mahon BP. Cell contact, prostaglandin $\mathrm{E}(2)$ and transforming growth factor beta 1 play non-redundant roles in human mesenchymal stem cell induction of CD4 + CD25(High) forkhead box P3+ regulatory T cells. Clin Exp Immunol. 2009;156:149-60.

25. Franquesa M, Hoogduijn MJ, Bestard O, Grinyo JM. Immunomodulatory effect of mesenchymal stem cells on B cells. Front Immunol. 2012;3:212.

26. Le Blanc K, Rasmusson I, Sundberg B, Gotherstrom C, Hassan M, Uzunel M, et al. Treatment of severe acute graft-versus-host disease with third party haploidentical mesenchymal stem cells. Lancet. 2004;363:1439-41.

27. Le Blanc K, Frassoni F, Ball L, Locatelli F, Roelofs H, Lewis I, et al. Mesenchymal stem cells for treatment of steroid-resistant, severe, acute graft-versus-host disease: a phase II study. Lancet. 2008;371:1579-86.

28. Lucchini G, Introna M, Dander E, Rovelli A, Balduzzi A, Bonanomi S, et al. Platelet-lysate-expanded mesenchymal stromal cells as a salvage therapy for severe resistant graft-versus-host disease in a pediatric population. Biol Blood Marrow Transplant. 2010;16:1293-301.

29. Le Blanc K, Frassoni F, Ball L, Locatelli F, Roelofs H, Lewis I, et al. Mesenchymal stem cells for treatment of steroid-resistant, severe, acute graft-versus-host disease: a phase II study. Lancet. 2008;371:1579-86.

30. Baker M. Stem-cell drug fails crucial trials. Nature. 2009. doi:10.1038/news.2009.894

31. Syed BA, Evans JB. Stem cell therapy market. Nat Rev Drug Discov. 2013;12:185-6.

32. Ciccocioppo R, Bernardo ME, Sgarella A, Maccario R, Avanzini MA, Ubezio C, et al. Autologous bone marrow-derived mesenchymal stromal cells in the treatment of fistulising Crohn's disease. Gut. 2011;60:788-98.

33. Duijvestein M, Vos AC, Roelofs $H$, Wildenberg ME, Wendrich $\mathrm{BB}$, Verspaget $\mathrm{HW}$, et al. Autologous bone marrowderived mesenchymal stromal cell treatment for refractory luminal Crohn's disease: results of a phase I study. Gut. 2010;59:1662-9.

34. Koh YG, Choi YJ. Infrapatellar fat pad-derived mesenchymal stem cell therapy for knee osteoarthritis. Knee. 2012;19:902-7.

35. Liang J, Li X, Zhang H, Wang D, Feng X, Wang H, et al. Allogeneic mesenchymal stem cells transplantation in patients with refractory RA. Clin Rheumatol. 2012;31:157-61.

36. Wang L, Cong X, Liu G, Zhou J, Bai B, Li Y, et al. Human umbilical cord mesenchymal stem cell therapy for patients with active rheumatoid arthritis: safety and efficacy. Stem cells Dev. 2013;22:3192-202.

37. Kim HY, Kim H, Oh KW, Oh SI, Koh SH, Baik W, et al. Biological markers of mesenchymal stromal cells as predictors of response to autologous stem cell transplantation in patients with amyotrophic lateral sclerosis: an investigator-initiated trial and in vivo study. Stem Cells. 2014;32:2724-31.

38. Krampera M, Cosmi L, Angeli R, Pasini A, Liotta F, Andreini A, et al. Role for interferon-gamma in the immunomodulatory activity of human bone marrow mesenchymal stem cells. Stem Cells. 2006;24:386-98.

39. Polchert D, Sobinsky J, Douglas G, Kidd M, Moadsiri A, Reina E, et al. IFN-gamma activation of mesenchymal stem cells for treatment and prevention of graft versus host disease. Eur J Immunol. 2008;38:1745-55.

40. Marigo I, Dazzi F. The immunomodulatory properties of mesenchymal stem cells. Semin Immunopathol. 2011;33:593-602.

41. Li W, Ren G, Huang Y, Su J, Han Y, Li J, et al. Mesenchymal stem cells: a double-edged sword in regulating immune responses. Cell Death Differ. 2012;19:1505-13.

42. Waterman RS, Tomchuck SL, Henkle SL, Betancourt AM. A new mesenchymal stem cell (MSC) paradigm: polarization into a pro-inflammatory MSC1 or an Immunosuppressive MSC2 phenotype. PLoS One. 2010;5:e10088.

43. Curti A, Trabanelli S, Salvestrini V, Baccarani M, Lemoli RM. The role of indoleamine 2,3-dioxygenase in the induction of immune tolerance: focus on hematology. Blood. 2009;113:2394-401.

44. Zhang J, Zhou S, Zhou Y, Feng F, Wang Q, Zhu X, et al. Hepatocyte growth factor gene-modified adipose-derived mesenchymal stem cells ameliorate radiation induced liver damage in a rat model. PLoS One. 2014;9:e114670.

45. Payne NL, Dantanarayana A, Sun G, Moussa L, Caine S, McDonald C, et al. Early intervention with gene-modified mesenchymal stem cells overexpressing interleukin-4 enhances antiinflammatory responses and functional recovery in experimental autoimmune demyelination. Cell Adhes Migr. 2012;6:179-89.

46. Chen Y, Song Y, Miao H, Xu Y, Lv M, Wang T, et al. Gene delivery with IFN-gamma-expression plasmids enhances the therapeutic effects of MSCs on DSS-induced mouse colitis. Inflamm Res. 2015;64:671-81.

47. Min CK, Kim BG, Park G, Cho B, Oh IH. IL-10-transduced bone marrow mesenchymal stem cells can attenuate the severity of acute graft-versus-host disease after experimental allogeneic stem cell transplantation. Bone Marrow Transplant. 2007;39:637-45.

48. Park MJ, Park HS, Cho ML, Oh HJ, Cho YG, Min SY, et al. Transforming growth factor beta-transduced mesenchymal stem cells ameliorate experimental autoimmune arthritis through reciprocal regulation of Treg/Th17 cells and osteoclastogenesis. Arthritis Rheum. 2011;63:1668-80.

49. Duijvestein M, Wildenberg ME, Welling MM, Hennink S, Molendijk I, van Zuylen VL, et al. Pretreatment with interferon-gamma enhances the therapeutic activity of mesenchymal stromal cells in animal models of colitis. Stem Cells. 2011;29:1549-58.

50. Chen H, Min XH, Wang QY, Leung FW, Shi L, Zhou Y, et al. Pre-activation of mesenchymal stem cells with TNF-alpha, IL1 beta and nitric oxide enhances its paracrine effects on radiationinduced intestinal injury. Sci Rep. 2015;5:8718.

51. Lan YW, Choo KB, Chen CM, Hung TH, Chen YB, Hsieh CH, et al. Hypoxia-preconditioned mesenchymal stem cells attenuate bleomycin-induced pulmonary fibrosis. Stem cell Res Therapy. 2015;6:97.

52. Kim HS, Shin TH, Lee BC, Yu KR, Seo Y, Lee S, et al. Human umbilical cord blood mesenchymal stem cells reduce colitis in mice by activating NOD2 signaling to COX2. Gastroenterology. 2013;145(1392-1403):e1391-8.

53. Lim JY, Park MJ, Im KI, Kim N, Jeon EJ, Kim EJ, et al. Combination cell therapy using mesenchymal stem cells and regulatory T-cells provides a synergistic immunomodulatory effect associated with reciprocal regulation of TH1/TH2 and th17/treg cells in a murine acute graft-versus-host disease model. Cell Transplant. 2014;23:703-14. 
54. Im KI, Park MJ, Kim N, Lim JY, Park HS, Lee SH, et al. Induction of mixed chimerism using combinatory cell-based immune modulation with mesenchymal stem cells and regulatory T cells for solid-organ transplant tolerance. Stem Cells Dev. 2014;23:2364-76.

55. Lee JH, Jeon EJ, Kim N, Nam YS, Im KI, Lim JY, et al. The synergistic immunoregulatory effects of culture-expanded mesenchymal stromal cells and CD4(+)25(+)Foxp3+ regulatory T cells on skin allograft rejection. PLoS One. 2013;8:e70968.
56. Lee ES, Lim JY, Im KI, Kim N, Nam YS, Jeon YW, et al. Adoptive transfer of Treg cells combined with mesenchymal stem cells facilitates repopulation of endogenous Treg cells in a murine acute GVHD model. PLoS One. 2015;10:e0138846.

57. Krampera M, Galipeau J, Shi Y, Tarte K, Sensebe L, Therapy, MSCCotISfC. Immunological characterization of multipotent mesenchymal stromal cells-The International Society for Cellular Therapy (ISCT) working proposal. Cytotherapy. 2013;15:1054-61. 